American Journal of Applied Sciences 6 (4): 726-735, 2009

ISSN 1546-9239

(C) 2009 Science Publications

\title{
Durability Assessment of Cylinder Block for Two Stroke Free Piston Linear Engine using Random Loading
}

\author{
${ }^{1}$ M.M. Rahman, ${ }^{2}$ A.K. Ariffin, ${ }^{2}$ S. Abdullah, ${ }^{1}$ M.M. Noor and ${ }^{1}$ Rosli A.B \\ ${ }^{1}$ Faculty of Mechanical Engineering, Automotive Excellence Center, \\ Universiti Malaysia Pahang Locked Bag 12, 25000 Kuantan, Pahang, Malaysia \\ ${ }^{2}$ Department of Mechanical and Materials Engineering, Faculty of Engineering, \\ Universiti Kebangsaan Malaysia, 43600 UKM, Bangi, Selangor, Malaysia
}

\begin{abstract}
This research presents the durability assessment of cylinder block for a two-stroke free piston engine using narrow band approach. The life prediction results are worthy for improving the component design at the very early developing stage. This approach is adequate for periodic loading, however, requires very large time records to accurately describe random loading processes. Fatigue damage in conventionally determined from time signals of the loading, frequently in the form of stress and strain. However, there are scenarios when a spectral form of loading is more appropriate. In this case the loading is defined in terms of its magnitude at different frequencies in the form of a Power Spectral Density (PSD) plot. The vibration fatigue calculation can be utilized where the random loading and response are categorized using power spectral density functions and the dynamic structure is modeled as a linear transfer function. This research also investigates the effect of the mean stress on the fatigue life prediction using the random loading. The acquired results indicate that the Goodman mean stress correction method gives the most conservative results with the Gerber and no (zero) mean stress method. It is observed that the compressive mean stresses are beneficial while the tensile mean stresses are detrimental to the fatigue life. The proposed technique is capable of determining premature products failure phenomena. Therefore, this technique is able to reduce cost, time to market, improve the product reliability and finally the user confidence.
\end{abstract}

Key words: Durability, cylinder block, random loading, finite element analysis, narrow band approach

\section{INTRODUCTION}

Durability is one of the most important design requirements that are essential for a new engine product to achieve successful market competitiveness. For the vehicle body structure the durability assessment has traditionally been performed at the later part of the product development stage when prototypes are available and heavily relied upon the result of the ground durability tests. This process is very time consuming and often results in over-design with weight penalties, which is the major obstacle to achieve fuel economy. Due to the development in the computeraided engineering (CAE) tools, a three-step process that includes multibody dynamic analysis, finite element analysis and fatigue life prediction, is widely used today for early product durability evaluation ${ }^{[1-3]}$. This approach helps the designer to acquire the necessary information for continuous design analysis and predicts the durability of the free piston linear generator engine in the early design stage, thereby eliminating some of the shortcomings in the traditional durability evaluation method.

Structures and mechanical components are frequently subjected to the oscillating loads which are random in nature. Random vibration theory has been introduced for more then three decades to deal with all kinds of random vibration behavior. Since fatigue is one of the primary causes of the component failure, fatigue life prediction has become a most important issue in almost any random vibration related problem $^{[4,5]}$. Nearly all structures or components have been designed using the time based structural and fatigue analysis methods. However, by developing a frequency based fatigue analysis approach, the accurate composition of the random stress or strain responses can be retained within a greatly optimized fatigue design process.

A vibration analysis is generally carried out to ensure that structural natural frequencies or resonant

Corresponding Author: M.M. Rahman, Faculty of Mechanical Engineering, University Malaysia Pahang, P.O. Box 12, 25000 Kuantan, Pahang, Malaysia Tel: +6-09-5492207 Fax: +6-09-5492244 
modes are not excited by the frequencies present in the applied load. Sometimes this is not possible and designers then have to estimate the maximum response at the resonance caused by the loading. These are the best performed in the frequency domain using the power spectral density functions of input loading and stress response. It is often easier to obtain a PSD of stress rather than a time history ${ }^{[6,7]}$.

The durability of the engine structure has increasingly vital issues due to the multidisciplinary research problem that involves the multibody dynamics, structural analysis and fatigue life prediction. Moreover, due to the requirement of the peak-valley editing and rainflow counting procedure in the fatigue life prediction, an analytical relationship between the dynamic stress and the fatigue life can not be obtained easily. The objectives of this research are to predict the fatigue life of cylinder block for free piston linear engine using narrow band approach and also to investigate the effect of mean stress on the fatigue life.

\section{ANALYSIS TECHNIQUES}

Durability analysis technique: Durability analysis can be used to determine how long a component can survive in a given service environment. In a general case the durability refers to the ability of a component to function in the presence of defects for a given environment/loading. In practice, however, the predominant failure mode is fatigue and hence, the term durability analysis will be used to describe the analysis of a fatigue performance. Fatigue analysis has traditionally been performed at a later stage of the design cycle. This is due to the fact that the loading information could only be derived from the direct measurements, which require a prototype ${ }^{[8]}$. The purpose of analyzing a structure early in the design cycle is to reduce the development time and cost. This is achieved for determining the critical regions of a structure and improving the design before prototypes are built and tested. Three computational processes are utilized to perform the durability analysis using CAE tools.

The finite element (FE) based durability analysis can be considered as a complete engineering analysis for the components. The fatigue life can be estimated for every element in the finite element model and the contour plots of life or damage can be obtained. The geometry information is provided by the FE results for each load case applied independently. Appropriate material properties are also provided for the desired fatigue analysis method. An integrated approach to durability analysis combines the multibody dynamic

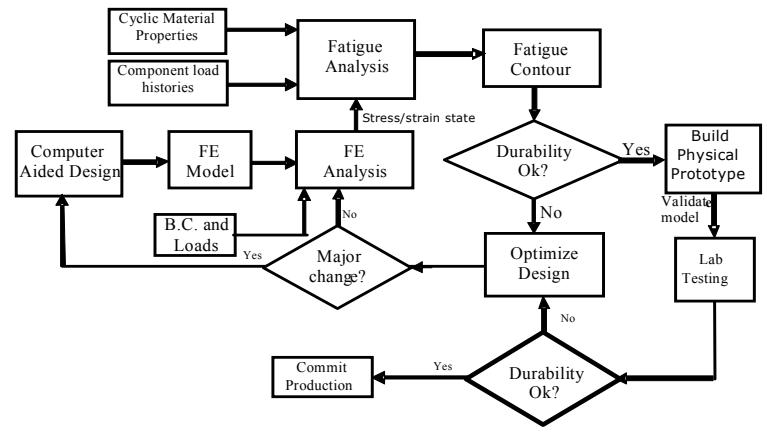

Fig. 1: The finite element based durability analysis

analysis, finite element analysis and the fatigue analysis into a consistent entity for the prediction of the durability of a component. The process of finite element based integrated durability analysis is shown in Fig. 1.

Narrow band approach: The stress power spectra density represents the frequency domain approach input into the fatigue ${ }^{[6]}$. This is a scalar function that describes how the power of the time signal is distributed among frequencies ${ }^{[9]}$. Mathematically this function can be obtained by using a Fourier Transform of the stress time history's auto-correlation function and its area represents the signal's standard deviation. It is clear that PSD is the most complete and concise representation of a random process.

Bendat ${ }^{[9]}$ has proposed first significant step towards a method of determining fatigue life from PSDs. Bendat showed that the PDF of the peaks for a narrow band signal tended towards Rayleigh distributions as the bandwidth reduced. Furthermore, for a narrow band time history Bendat assumed that all the positive peaks in the time history would be followed by corresponding troughs of similar magnitude regardless of weather they actually formed stress cycles. Using this assumption, the PDF of stress range would also to a Rayleigh distribution. Bendat used a series of equations derived by Race ${ }^{[10]}$ to estimate the expected number of peaks using moments of area beneath the PSD.

Assume $\mathrm{p}(\mathrm{S})$ is the Rayleigh distribution which $\mathrm{s}$ represented by a narrow band process and stress amplitude, $\mathrm{S}$, can be treated as a continuous random variable.

$$
\mathrm{p}(\mathrm{S})_{\mathrm{NB}}=\mathrm{f}\left(\mathrm{m}_{0}\right)=\frac{\mathrm{S}}{4 \mathrm{~m}_{0}} \mathrm{e}^{\frac{-\mathrm{s}^{2}}{\mathrm{~m}_{0}}}
$$

The expected total fatigue damage for narrow band Gaussian process, 


$$
\begin{aligned}
E[D] & =\sum_{i} \frac{n_{i}}{N\left(S_{i}\right)}=\frac{S_{t}}{K} \int S^{b} p(S) d S \\
& =\frac{E[P] T}{K} \int S^{b}\left[\frac{S}{4 m_{0}} e^{\frac{-S^{2}}{8 m_{0}}}\right] d S
\end{aligned}
$$

where $N\left(S_{i}\right)$ is the number of cycles of stress range $S$ occurring in $T$ seconds, $n_{i}$ is the actual counted number of cycle, $S_{t}$ is the total number of cycles equals to $\mathrm{E}[\mathrm{P}] \mathrm{T}$.

Even though any statistical model of $\mathrm{S}$ can be employed, it is common to use the Weibull distribution

$$
F(S)=1-e^{-\left(\frac{s}{\alpha}\right)^{\beta}}
$$

where $\alpha$ and $\beta$ are the scale parameter or characteristic life and shape parameter or Weibull slope respectively. For Weibull distribution

$$
\mathrm{E}\left(\mathrm{S}^{\mathrm{b}}\right)=\alpha^{\mathrm{b}} \Gamma\left(\frac{\mathrm{b}}{\beta}+1\right)
$$

where $\Gamma(\cdot)$ is the Gamma function.

The Weibull distribution reduces to Rayleigh distribution when $\beta=2$. Rayleigh is the distribution of peaks or ranges or amplitudes in a stationary narrow band Gaussian process that has an RMS value.

$$
\alpha=\sqrt{2} \mathrm{RMS}=\sqrt{2 \mathrm{~m}_{0}}
$$

Therefore, we get solution from Eq. (3) is expressed in Eq. (5)

$$
\mathrm{E}\left(\mathrm{S}^{\mathrm{b}}\right)=\left(\sqrt{2 \mathrm{~m}_{0}}\right)^{\mathrm{b}} \Gamma\left(\frac{\mathrm{b}}{\beta}+1\right)
$$

The expected total fatigue damage, E[D], of the zero mean stationary narrow band Gaussian process over a time interval $\tau$ can be expressed as Eq. (6).

$$
\mathrm{E}[\mathrm{D}]=\frac{\mathrm{E}[\mathrm{P}] \mathrm{T}}{\mathrm{K}}\left(\sqrt{2 \mathrm{~m}_{0}}\right)^{\mathrm{b}} \Gamma\left(\frac{\mathrm{b}}{\beta}+1\right)
$$

This is the first frequency response method for predicting fatigue damage from PSDs and it assumes that the PDF of peaks is equal to the PDF of stress amplitudes. The narrow band solution was then obtained by substitutions the Rayleigh PDF of peaks with the PDF of stress ranges. The full equation is

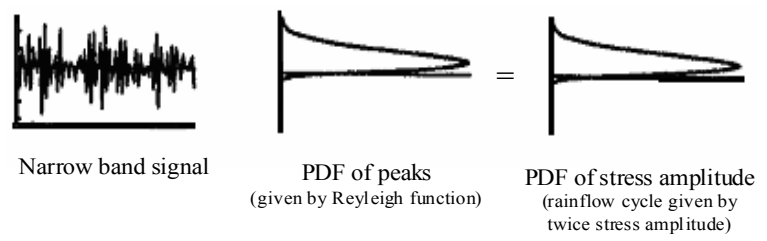

Fig. 2: Basis of the narrow band solution ${ }^{[6]}$

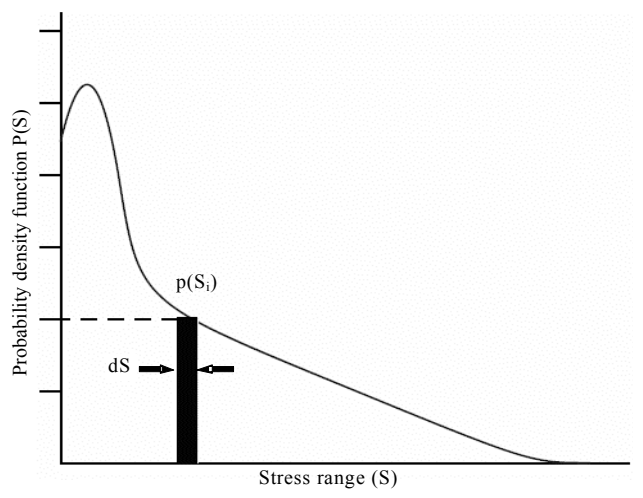

Fig. 3: Probability density functions

obtained by noting that $S_{t}$ is equal to $E[P]$.T, where $T$ is the life of the structure in seconds. The basis of the narrow band solution is shown in Fig. 2.

The most convenient way of storing stress range histogram information is in the form of a probability density function (PDF) of stress ranges ${ }^{[1]}$. A typical representation of this function is shown in Fig. 3. It is easily to transform from a stress range histogram to a PDF, or back to time histories. The bin widths used and the total number of cycles recorded in the histogram are the only additional information required. To get a PDF from a rainflow histogram each bin in the rainflow count has to be multiplied by $S_{t} \times d S$, where $S_{t}$ is the total number of cycles in histogram and dS is the interval width. The probability of the stress range occurring between $\mathrm{S}_{\mathrm{i}}-\mathrm{dS} / 2$ and $\mathrm{S}_{\mathrm{i}}+\mathrm{dS} / 2$ is given by $\mathrm{p}\left(\mathrm{S}_{\mathrm{i}}\right) \mathrm{dS}$. The actual counted number of cycles, $n_{i}=p(S) d S S_{t}$. The allowable number of cycles,

$$
\mathrm{N}\left(\mathrm{S}_{\mathrm{i}}\right)=\frac{\mathrm{k}}{\mathrm{S}^{\mathrm{b}}}
$$

Then damage is defined as,

$$
E[D]=\sum_{i} \frac{n_{i}}{N\left(S_{i}\right)}=\frac{S_{t}}{k} \int S^{b} p(S) d S
$$

Failure occurs, $\mathrm{D} \geq 1.0$. 
Am. J. Appl. Sci., 6 (4): 726-735, 2009

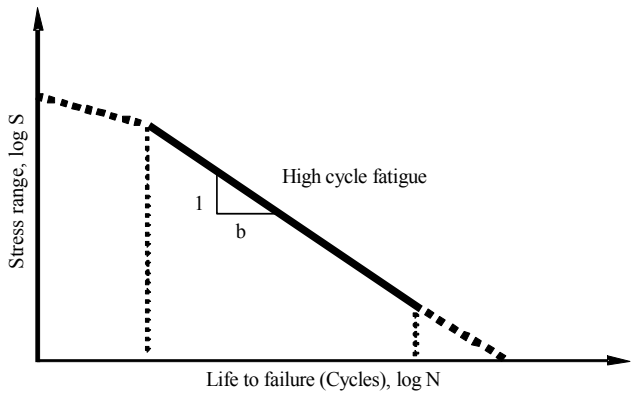

Fig. 4: Typical S-N curve for high cycle fatigue

In order to compute fatigue damage over the lifetime of the structure in seconds the form of materials S-N data must also be defined using the material parameters $\mathrm{k}$ and $\mathrm{b}$. The typical S-N curve for high cycle fatigue is as shown in Fig. 4. This figure simply shows that, under constant amplitude cyclic loading, a linear relationship exists between cycles to failure $(\mathrm{N})$ and applied stress range $(\mathrm{S})$ when plotted on $\log -\log$ research. There are two alternative ways of defining this relationship, as given in Eq. (9).

$$
\mathrm{N}=\mathrm{kS}^{-\mathrm{b}}
$$

where,

$$
\mathrm{b}=-\frac{1}{\mathrm{~b} 1} \mathrm{and} \mathrm{k}=(\mathrm{SRI} 1)^{\mathrm{b}}
$$

A method for computing PSD moments is described by Rahman ${ }^{[1]}$. Some very important statistical parameters can be computed from these moments. These parameters are root mean square (RMS), number of Zero crossing with positive slope $(\mathrm{E}[0])$, number of peaks per second $(\mathrm{E}[\mathrm{P}])$. The formulas in Eq. (10) highlight these properties of the spectral moments.

$$
\mathrm{RMS}=\sqrt{\mathrm{m}_{0}}, \mathrm{E}[0]=\sqrt{\frac{\mathrm{m}_{2}}{\mathrm{~m}_{0}}}, \mathrm{E}[\mathrm{P}]=\sqrt{\frac{\mathrm{m}_{4}}{\mathrm{~m}_{2}}}
$$

where $\mathrm{m}_{0}, \mathrm{~m}_{1}, \mathrm{~m}_{2}$ and $\mathrm{m}_{4}$ are the zero, $1^{\text {st }}, 2^{\text {nd }}$ and $4^{\text {th }}$ order moment of area of the PSD respectively.

Another important property of spectral moments is the fact that it is possible to express the irregularity factor as a function of the zero, second and fourth order spectral moments, as shown in Eq. (11).

$$
\gamma=\frac{\mathrm{E}[0]}{\mathrm{E}[\mathrm{P}]}=\frac{\mathrm{m}_{2}}{\sqrt{\mathrm{m}_{0} \mathrm{~m}_{4}}}
$$

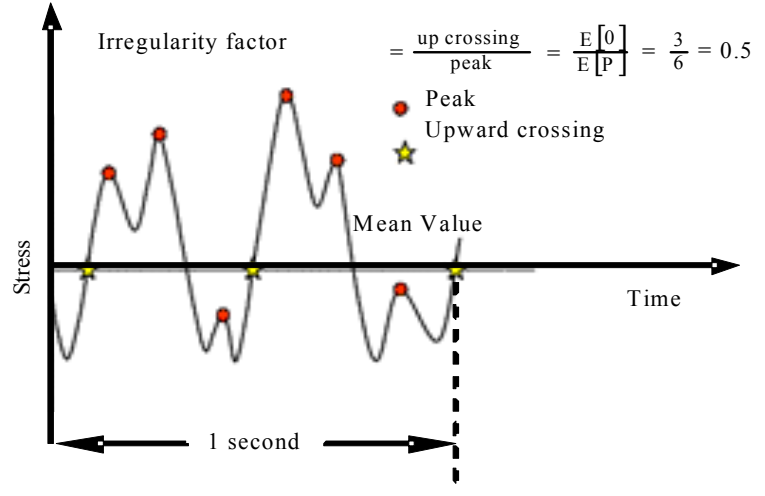

Fig. 5: Calculation of the irregular factor, $\gamma$

Figure 5 shows how to calculate the irregularity factor. When irregularity factor is equal to 0 , there are an infinite number of peaks for every zero upcrossing. This is considered a wide band random process. The value of irregularity factor is equal to 1 corresponds to one peak per one zero upcrossing and it represents a narrow-band random process. Alternatively, a narrowband process can be defined by the width of its spectrum. For this reason, the spectral width parameter, $\lambda$, is introduced as

$$
\lambda=\sqrt{1-\gamma^{2}}
$$

That means $\lambda=0$ represents a narrow-band random process.

The irregularity factor $\gamma$ is an important parameter that can be used to evaluate the concentration of the process near a central frequency. Therefore, the irregularity factor can be used to determinate whether the process is narrow band or wide band. Irregularity factor varies between 1 and 0 . A narrow band process $(\gamma \rightarrow 1)$ is characterized by only one predominant central frequency indicating that the number of peaks per second is very similar to the number of zero crossings of the signal. This assumption leads to the fact that the PDF of the fatigue cycles range is the same as the PDF of the peaks in the signal. In this case fatigue life is easy to estimate.

Loading information: Several types of the variable amplitude loading histories were selected from the Society of Automotive Engineers (SAE) and American Society for Testing and Materials (ASTM) profiles for the FE based fatigue analysis. It is important to emphasize that these sequences are not indented to represent standard loading spectra in the same way that Carlos or Falstaf ${ }^{[11]}$ was performed. However, they 
do
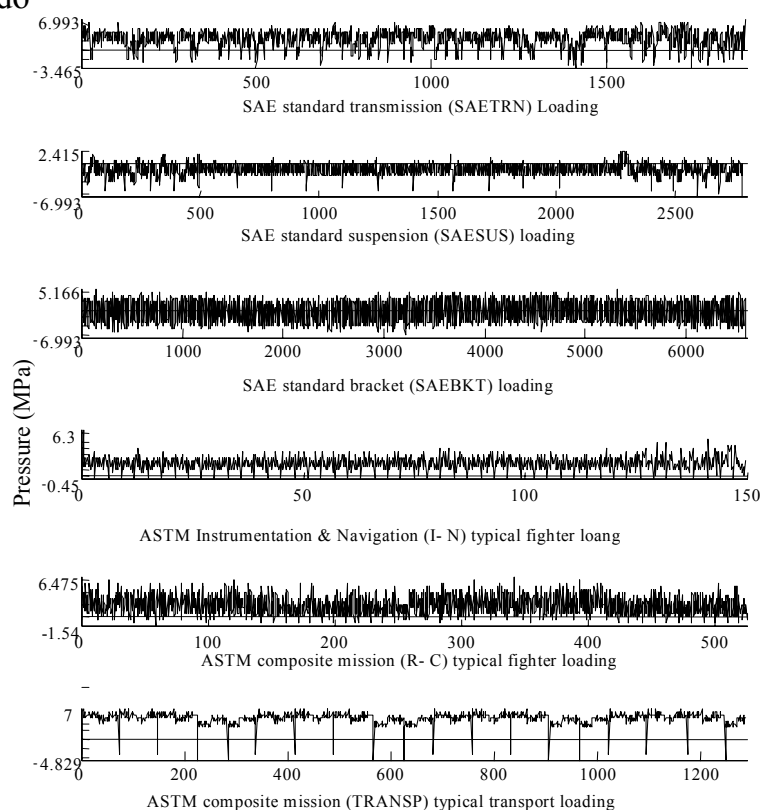

Time (Seconds)

Fig. 6: Different time loading histories

contain many features which are typical of the automotive industries applications and therefore, are useful in the evaluation of the life estimation methods. The component was loaded with three random time histories, corresponding to typical histories for the transmission, suspension and bracket components at different load levels. The first load history has a predominantly tensile (positive) mean which reflects sudden changes in mean, which is referred to as the transmission history. The second load history has a predominantly compressive (negative) mean, which is referred as suspension history. The third load history representing a vibration with nearly zero mean loads, which is referred as the bracket history. The detailed information about these histories can be referred in the literature ${ }^{[1,12]}$. These histories were scaled to two peak strain levels and used as full-length histories. In addition, a random history including many spikes was selected for the simulation of spike removal. The variable amplitude load-time histories are shown in Fig. 6 and the corresponding power spectral densities responses are also shown in Fig. 7. The terms of SAETRN, SAESUS and SAEBKT represent the loadtime history for the transmission, suspension and bracket respectively. The considered load-time histories are based on the SAE's profile. In addition, I-N, R-C and TRANSP are representing the ASTM instrumentation and navigation typical fighter, ASTM composite mission typical fighter loading history and
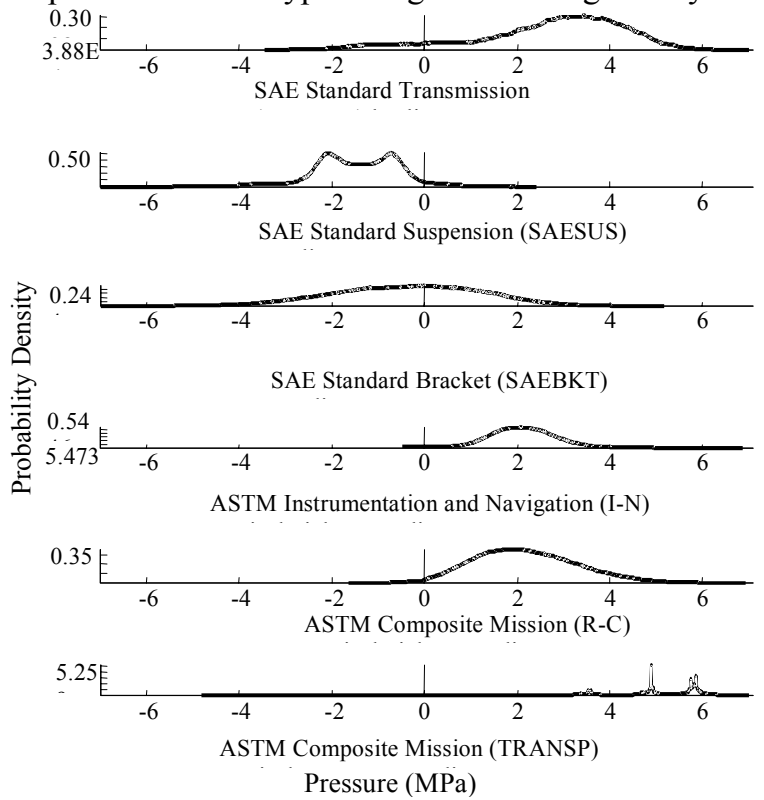

Fig. 7: Power spectral densities response

ASTM composite mission typical transport loading history respectively ${ }^{[12]}$. The abscissa is the time, in seconds.

\section{RESULTS AND DISCUSSION}

Finite element modeling and analysis: A geometric model of the cylinder block of the free piston engine is considered in this study. Three-dimensional model geometry was developed in CATIA ${ }^{\circledR}$ software. Since the tetrahedral is found to be the best meshing technique, the 4 nodes tetrahedral (TET4) element version of the cylinder block was used for the initial analysis. In addition, the TET4 compared to the 10 nodes tetrahedral (TET10) element mesh using the same global mesh length for the highest loading conditions $(7.0 \mathrm{MPa})$ in the combustion chamber. The investigating the results, it can be found that the TET10 mesh predicted higher von Mises stresses than that the TET4 mesh. The TET10 mesh is presumed to represent a more accurate solution since TET4 meshes are known to be dreadfully stiff ${ }^{[13]}$. TET4 employed a linear order interpolation function while TET10 used quadratic order interpolation function. For the same element size, the TET10 is expected to be able to capture the high stress concentration associated with the bolt holes. A TET10 was then finally used for the solid mesh. Mesh study is performed on FE model to ensure 


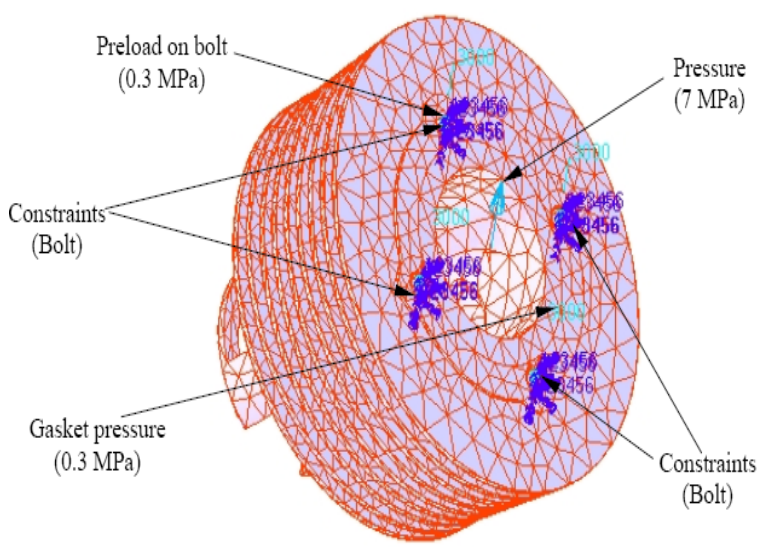

Fig. 8: Loading and constraints on the cylinder block

sufficiently fine sizes are employed for accuracy of calculated results but at competitive cost (CPU time). In the process, specific field variable is selected and its convergence is monitored and evaluated. Sensitivity analysis was performed to obtain the optimum element size. The analysis was performed iteratively at different element lengths until the solution obtained appropriate accuracy. Convergence of the stresses was observed, as the mesh size was successively refined. The element size of $0.20 \mathrm{~mm}$ was finally considered. A total of 35415 elements and 66209 nodes were generated with $0.20 \mathrm{~mm}$ element length. Compressive loads were applied as pressure ( $7 \mathrm{MPa}$ ) acting on the surface of the combustion chamber and preloads were applied as pressure $(0.3 \mathrm{MPa})$ acting on the bolt-hole surfaces. In addition, preload was also applied on the gasket surface generating pressure of $0.3 \mathrm{MPa}$. The loading and constraints on the cylinder block are shown in Fig. 8. The constraints were applied on the bolt-hole for all six degree of freedoms.

Stress analysis: The finite element results of time domain (Pseudo-static method) i.e., the maximum principal stresses distribution is presented in Fig. 9. The fatigue life of time domain histories are performed using the stress-life method employed rainflow cycle counting technique ${ }^{[14,15]}$. Time domain fatigue approach consists of a number of steps. The first is to count the number of stress cycles in the response time history. This is performed through a process of rain flow cycle counting. Damage from each cycle is determined, typically from an S-N curve. The damage is then summed over all cycles using linear damage summation techniques to determine the total life. The frequency response analyses were performed using the finite element analysis code. The frequency response analysis

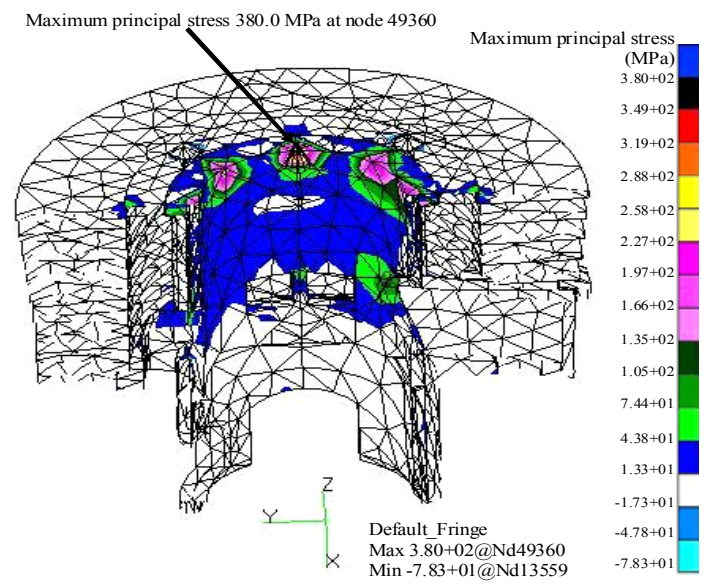

Fig. 9: Maximum principal stresses distribution for the Pseudo-static linear analysis

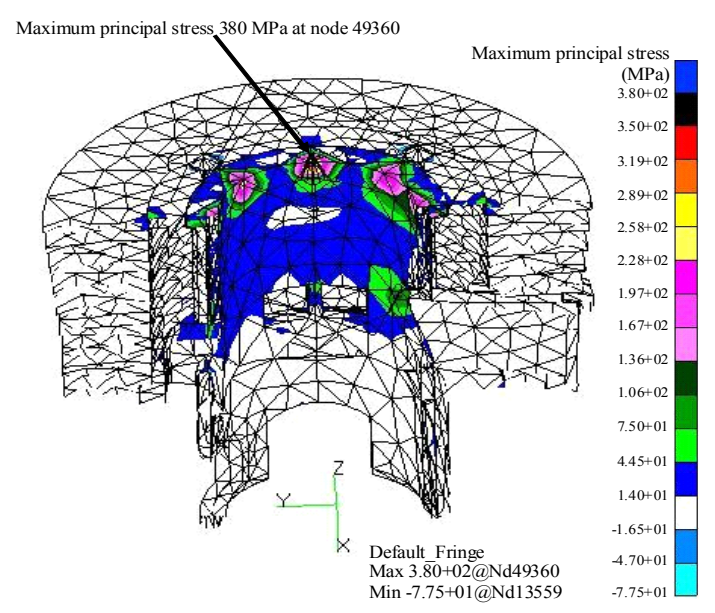

Fig. 10: Maximum principal stresses distribution for the frequency response analysis with zero $\mathrm{Hz}$

used the damping ratio of $5 \%$ of critical. The damping ratio is the ratio of the actual damping in the system to the critical damping. Most of the experimental modal reported that the modal damping in terms of nondimensional critical damping ratio expressed as a percentage ${ }^{[16,17]}$. In fact, most structures have critical damping values in the range of $0-10 \%$, with values of $1-5 \%$ as the typical range ${ }^{[18]}$ Zero damping ratio indicates that the mode is undamped. Damping ratio of one represents the critically damped mode. The result of the frequency response finite element analysis with zero $\mathrm{Hz}$ i.e. the maximum principal stresses distribution of the cylinder block is presented in Fig. 10 respectively. From the results, the maximum and minimum principal stresses of 380.0 and -78.3 MPa for 
Am. J. Appl. Sci., 6 (4): 726-735, 2009

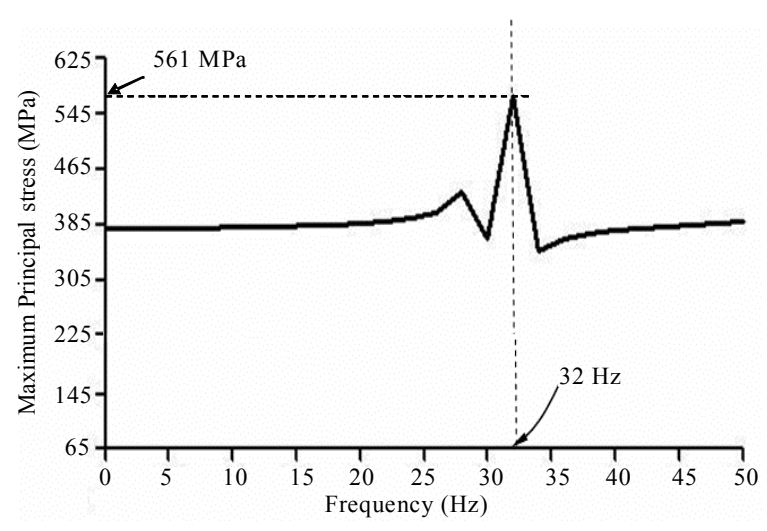

Fig. 11: Maximum principal stresses plotted against frequency

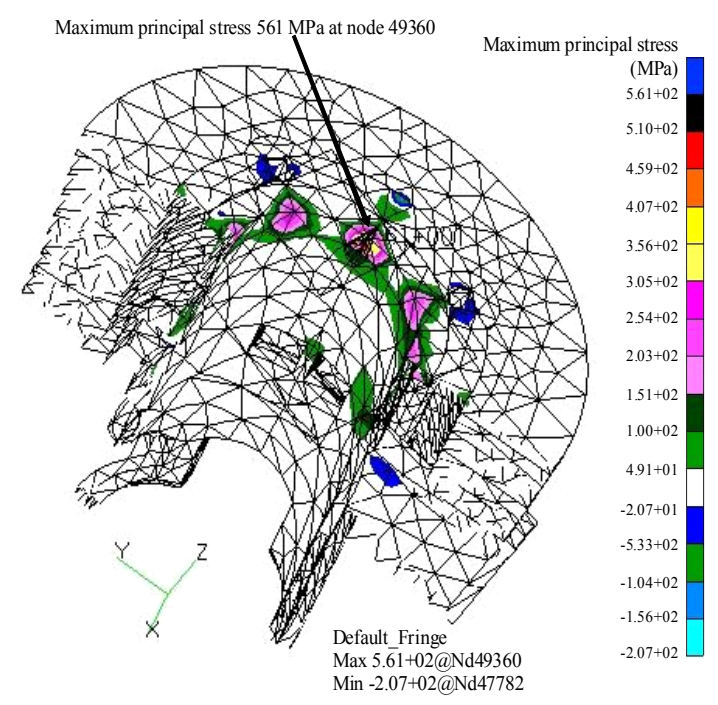

Fig. 12: Maximum principal stresses contour for the frequency response analysis with $32 \mathrm{~Hz}$

the Pseudo static analysis and 380.0 and $-77.5 \mathrm{MPa}$ for the frequency response analysis for zero $\mathrm{Hz}$ were obtained respectively. These two maximum principal stresses contour plots are almost identical.

The variation of the maximum principal stresses with the frequency is shown in Fig. 11. It can be seen that the maximum principal stress varies with the higher frequencies. This variation is due to the dynamic influences of the first mode shape. It is also observed that the maximum principal stress occurs at a frequency of $32 \mathrm{~Hz}$. The maximum principal stresses of the cylinder block for $32 \mathrm{~Hz}$ is presented in Fig. 12. From the results, the maximum and minimum principal stresses of 561.0 and $-207.0 \mathrm{MPa}$ were obtained at node 49360 and 47782 respectively.
Table 1: Results of the modal analysis

\begin{tabular}{lc}
\hline Mode No. & Natural frequency $(\mathrm{Hz})$ \\
\hline 1 & 186.32 \\
2 & 259.05 \\
3 & 306.61 \\
4 & 317.65 \\
5 & 327.65 \\
6 & 339.84 \\
7 & 382.20 \\
8 & 462.17 \\
9 & 650.87 \\
10 & 721.28 \\
\hline
\end{tabular}

Modal analysis: The modal analysis is usually used to determine the natural frequencies and mode shapes of a component. It can also be used as the starting point for the frequency response analysis. The finite element analysis codes usually used several mode extraction methods. The Lanczos mode extraction method is used in this study due to Lanczos is the recommended method for the medium to large models. In addition to its reliability and efficiency, the Lanczos method supports sparse matrix methods that significantly increase computational speed and reduce the storage space. This method also computes precisely the eigenvalues and eigenvectors. The number of modes was extracted and used to obtain the cylinder block stress histories, which is the most important factor in this analysis. Using this method to obtain the first 10 modes of the cylinder block, which are presented in Table 1 and the shape of the mode are shown in Fig. 13. It can be seen that the working frequency $(50 \mathrm{~Hz})$ is far away from the natural frequency $(186.32 \mathrm{~Hz})$ of the first mode.

Fatigue life prediction analysis: The results of the fatigue life contour for the SAETRN loading histories at most critical locations for Pseudo-static analysis and frequency response approach with $32 \mathrm{~Hz}$ are shown in Fig. 14 and 15 respectively. The minimum life prediction for Pseudo-static analysis and frequency response approach with $32 \mathrm{~Hz}$ are obtained $10^{7.67}$ and $10^{9.44}$ seconds respectively. It would be expected that the condition of lower stress would correspond to longer life and vice versa. However, the results indicates the opposite because the frequency resolution of the transfer function are selected the small value then the result show the non-conservative prediction. From Fig. 14 and 15, it can be seen that the fatigue life contours are different and most damage was found at frequency of $32 \mathrm{~Hz}$.

For the purpose of validation, the predicted life listed below will be taken to be the definitive answers. Table 2 shows the correlation between the frequency response analysis method and the conventional time 

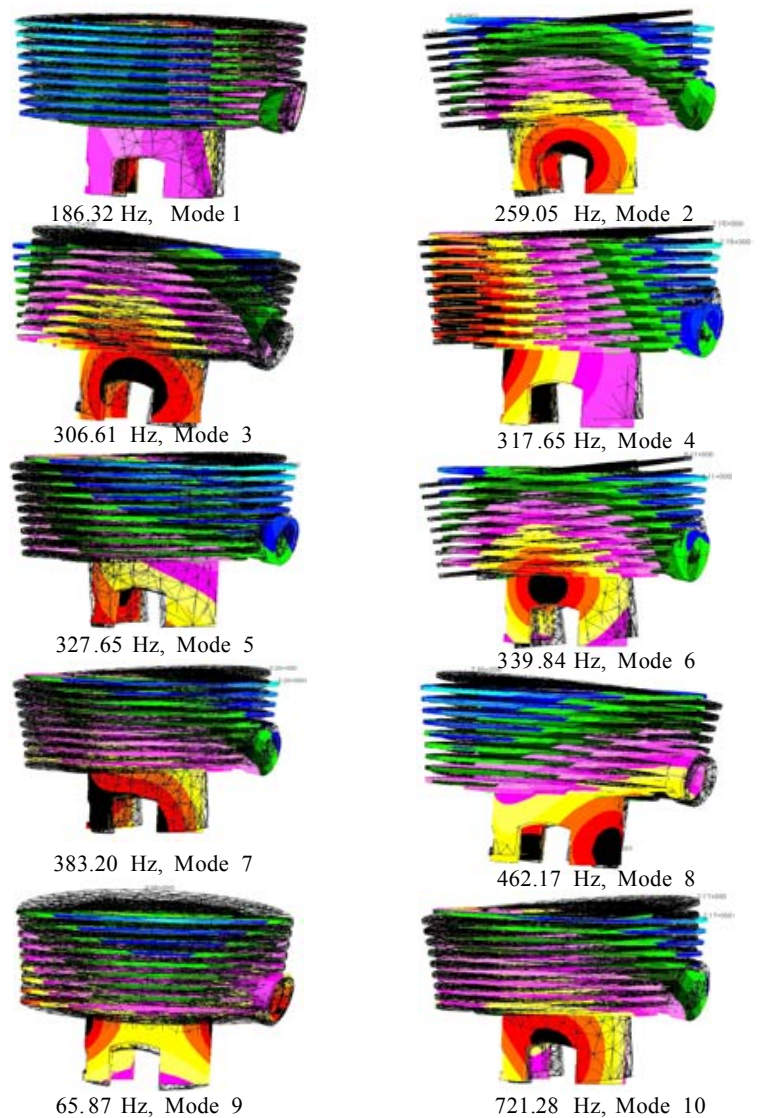

$317.65 \mathrm{~Hz}$, Mode 4

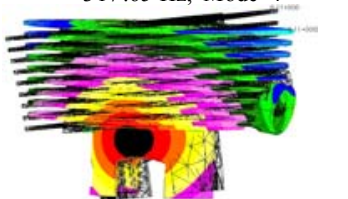

$339.84 \mathrm{~Hz}$, Mode 6

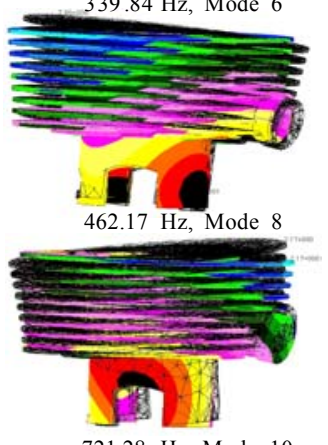

$721.28 \mathrm{~Hz}$, Mode 10

Fig. 13: The mode shapes of the cylinder block

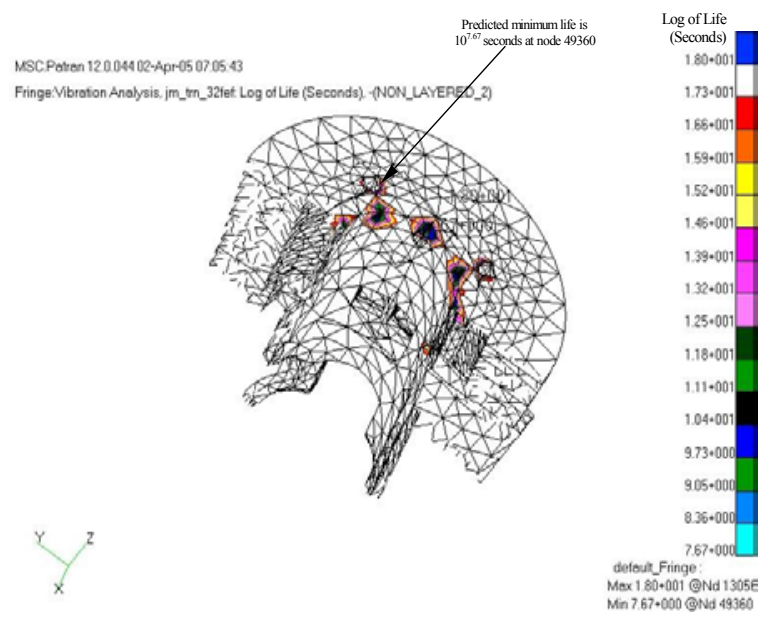

Fig. 14: Predicted fatigue life contours plotted using the pseudo-static method

domain Pseudo-static approach. The predicted fatigue life on log-log coordinates using the Pseudo-static and

Table 2:Predicted life in seconds between two approaches at most critical location (node 50420)

\begin{tabular}{|c|c|c|}
\hline \multirow[b]{2}{*}{$\begin{array}{l}\text { Loading } \\
\text { Conditions }\end{array}$} & \multicolumn{2}{|c|}{$\begin{array}{l}\text { Predicted fatigue life in seconds at } \\
\text { critical location (node 50420) }\end{array}$} \\
\hline & $\begin{array}{l}\text { Pseudo-static } \\
\text { approach (seconds) }\end{array}$ & $\begin{array}{l}\text { Vibration analysis } \\
\text { (seconds) }\end{array}$ \\
\hline SAETRN & $1.14 \times 10^{8}$ & $2.10 \times 10^{7}$ \\
\hline SAESUS & $6.34 \times 10^{9}$ & $8.74 \times 10^{10}$ \\
\hline SAEBKT & $7.56 \times 10^{7}$ & $4.06 \times 10^{8}$ \\
\hline ASTM I-N & $3.02 \times 10^{9}$ & $2.30 \times 10^{8}$ \\
\hline ASTM R-C & $1.27 \times 10^{6}$ & $6.02 \times 10^{7}$ \\
\hline ASTM TRANSP & $1.15 \times 10^{7}$ & $2.27 \times 10^{9}$ \\
\hline
\end{tabular}

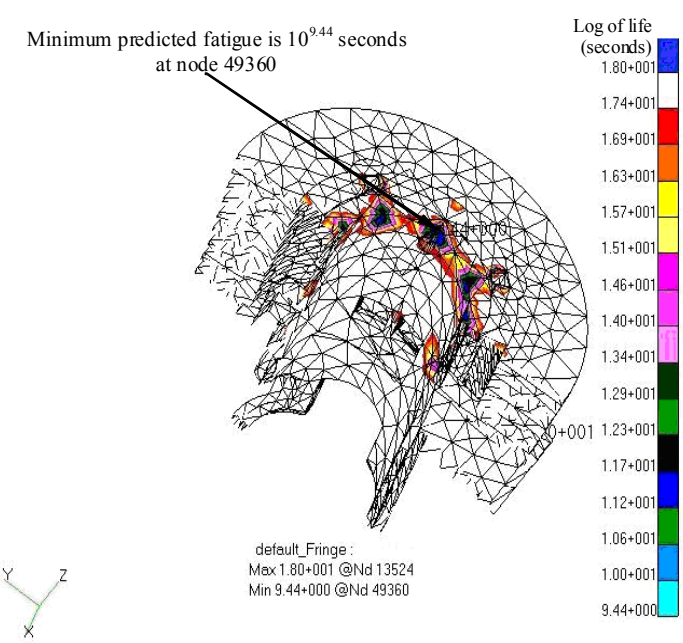

Fig. 15: Predicted fatigue life contours plotted using the frequency response analysis for $32 \mathrm{~Hz}$

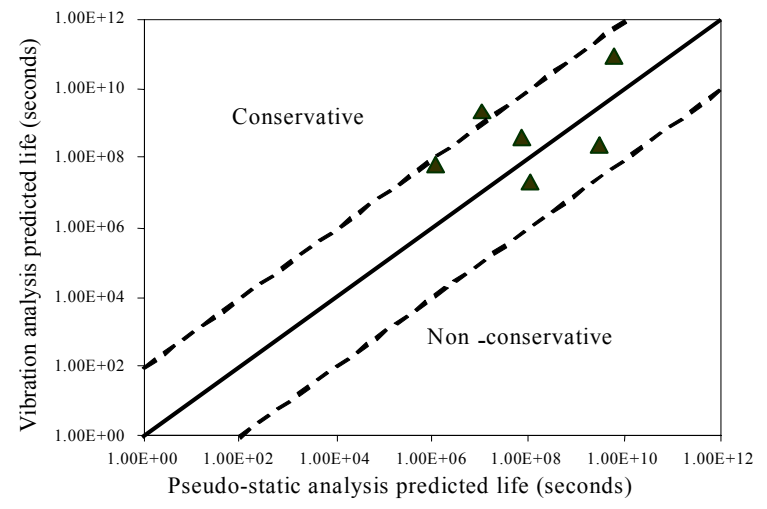

Fig. 16: Correlation between the fatigue life between the Pseudo-static and frequency response analysis

frequency response analysis is presented in Fig. 16. The solid straight line in Fig. 16 represents the perfect correlation between the pseudo-static and frequency 
response analysis results i.e. one to one correspondence if the vibration fatigue predicted life exactly equivalent to the pseudo-static predicted life. The two straight dotted lines represent a three times factor indicating a goodness band. Data points that fall above the solid line represent non-conservative estimates, while points below the solid line represent conservative predictions in comparison to the pseudo-static time domain results. It can be seen that the predicted fatigue life obtained from the vibration analysis using narrow band approach is in good agreement with the predicted life using the Pseudo-static time domain approach. Most of the predicted data falling well within the scatter band, which is shown in Fig. 16. It can be also seen that the frequency response analysis method tended to be conservative due to the data points concentrates on the lower band i.e., with the solid line and the lower dotted line. This indicates that the predicted life using the frequency response approach was reasonable and acceptable.

Effect of frequency resolution: Frequency resolution of the transfer function is significant to capture the input PSD. The significances of the frequency resolutions of SAETRN loading histories are also shown in Fig. 17 and 18. Two types of Fast Fourier Transform (FFT) buffer size width namely 8192:0.06104 and 16384:0.03052 $\mathrm{Hz}$ were used in these figures. The FFT buffer size defines the resolution of the power spectrum. The buffer must be a power of 2 and of course the longer the buffer, the higher the resolution of the spectral lines. To calculate the resolution divide the Nyquist frequency by half the FFT buffer size. If the Nyquist frequency is $250 \mathrm{~Hz}$ and the FFT buffer size selected is 8192 , then the spectral lines are $250 /(8192 / 2)=0.06104 \mathrm{~Hz}$ apart. Another use of a smaller buffer size is for short data files as these cannot be adequately analyzed with a big buffer, since there may not be enough data to give a good spectral average. Using a smaller buffer size could give a better spectral average at the expense of spectral line width. The total area under each input PSD curve is determined to be identical. However, the 16384:0.03052 Hz width has twice as many points compares to the $8192: 0.06104 \mathrm{~Hz}$.

The full set of the comparison results for the untreated polished cylinder block at critical location (node 49360) is given in Table 3 with various loading conditions. The narrow band solution is considered in this study. It is observed that from Table 3, the predominantly compressive mean loading (SAESUS) produces longer life than the tensile (positive) mean

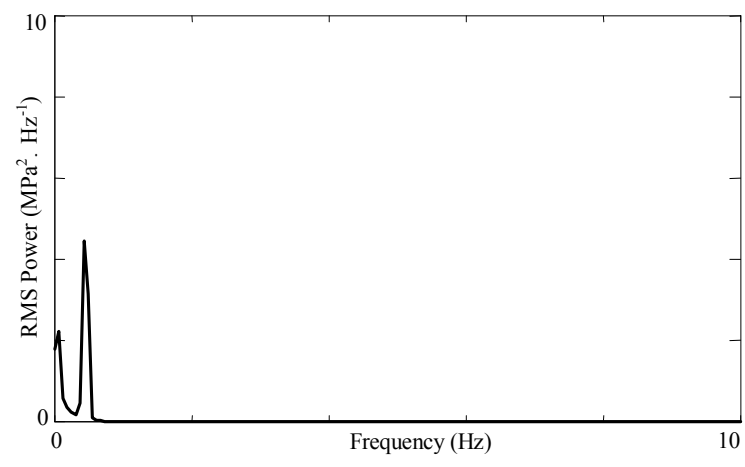

Fig. 17: Power spectral density at FFT buffer size of 16384:0.03052 Hz width

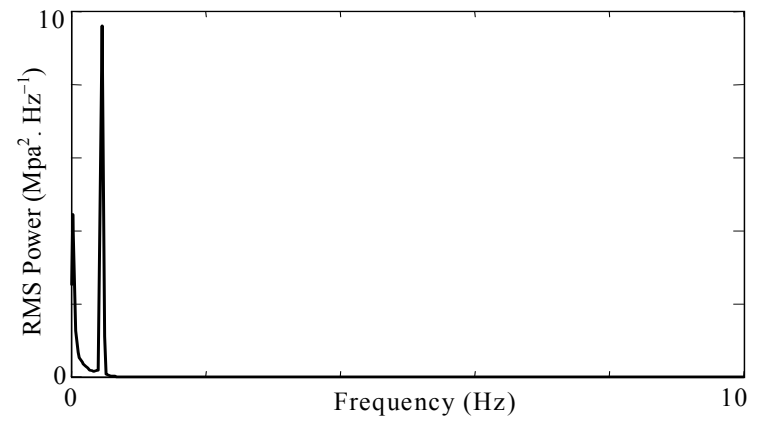

Fig. 18: Power spectral density at FFT buffer size of 8192:0.06104 Hz width

Table 3: Predicted life in seconds at critical location (node 49360) for various loading conditions and materials Predicted vibration fatigue life in seconds

\begin{tabular}{|c|c|c|c|c|c|c|}
\hline \multirow{2}{*}{$\begin{array}{l}\text { Loading } \\
\text { conditions }\end{array}$} & \multicolumn{3}{|l|}{ 2024-HV-T6 } & \multicolumn{3}{|l|}{ 6061-T6-80-HF } \\
\hline & No (zero) mean & Goodman & Gerber & No (zero) mean & Goodman & Gerber \\
\hline SAETRN & $2.75 \times 10^{9}$ & $2.53 \times 10^{9}$ & $2.74 \times 10^{9}$ & $2.10 \times 10^{7}$ & $2.02 \times 10^{7}$ & $2.09 \times 10^{7}$ \\
\hline SAESUS & $1.53 \times 10^{12}$ & $1.46 \times 10^{12}$ & $1.52 \times 10^{12}$ & $8.74 \times 10^{10}$ & $7.97 \times 10^{9}$ & $8.62 \times 10^{9}$ \\
\hline SAEBKT & $3.36 \times 10^{10}$ & $3.15 \times 10^{10}$ & $3.35 \times 10^{10}$ & $4.06 \times 10^{8}$ & $8.86 \times 10^{7}$ & $1.03 \times 10^{8}$ \\
\hline $\mathrm{I}-\mathrm{N}$ & $1.47 \times 10^{10}$ & $1.39 \times 10^{10}$ & $1.46 \times 10^{10}$ & $2.30 \times 10^{8}$ & $2.21 \times 10^{8}$ & $2.28 \times 10^{8}$ \\
\hline $\mathrm{R}-\mathrm{C}$ & $1.96 \times 10^{9}$ & $1.82 \times 10^{9}$ & $1.95 \times 10^{9}$ & $6.02 \times 10^{7}$ & $3.75 \times 10^{7}$ & $5.81 \times 10^{7}$ \\
\hline TRANSP & $1.51 \times 10^{11}$ & $1.44 \times 10^{11}$ & $1.50 \times 10^{11}$ & $2.27 \times 10^{9}$ & $2.12 \times 10^{9}$ & $2.24 \times 10^{9}$ \\
\hline
\end{tabular}


loading (SAETRN) due to the compressive mean stresses are beneficial to the fatigue life. In designing for the durability, the presence of a nonzero mean stress can influence the fatigue behavior of materials because a tensile or a compressive mean stress has been shown to be responsible for accelerating or decelerating crack initiation. It is observed that the compressive mean stresses are beneficial while the tensile mean stresses are detrimental to the fatigue life. This phenomenon is dominant when the mean stress levels are relatively low compared to the cyclic yield stress and the fatigue behavior falls in the long-life regime where elastic strain is dominant. The compressive mean loading beneficial for fatigue. In addition, the R-C loading condition gives the lowest lives for all materials.

\section{CONCLUSION}

The vibration fatigue analysis technique has presented when the random loading are categorized using PSD functions. Narrow band frequency response analysis has applied to a typical cylinder block for the new two-stroke free piston linear engine. According to the results, it can be concluded that Goodman mean stress correction method gives the most conservative prediction for all loading conditions and materials. The results clearly indicate that the AA2024-HV-T6 is a superior material for all the mean stress correction methods. The life predicted from the vibration fatigue analysis is consistently higher except for the bracket loading condition. The proposed approach can be used for durability assessment of a prototype engine with actual service conditions in the early developing stage.

\section{ACKNOWLEDGMENTS}

The authors would like to thank Department of Mechanical and Materials Engineering, Universiti Kebangsaan Malaysia for provides lab facilities. The authors are grateful to the University Malaysia Pahang for providing the financial support.

\section{REFERENCES}

1. Rahman, M.M., 2007. Finite element based durability assessment for a new free piston linear engine. Ph.D Thesis. Universiti Kebangsaan Malaysia.

2. Rahman, M.M., A.K. Ariffin, N. Jamaludin and C.H.C. Haron, 2007. Finite element based durability assessment of a free piston linear engine component. Structural Durability and Health Monitoring (SDHM), 3 (1): 1-13.
3. Rahman, M.M., A.K. Ariffin, N. Jamaludin and C.H.C. Haron, 2006. Durability Assessment of a New Free Piston Spark Ignition Engine: A Computational Approach. Journal of Technology Part-A, 45 (A): 81-102.

4. Soong, T.T. and M. Grigoriu, 1993. Random Vibration of Mechanical and Structural Systems. UK: Prentice Hall International Ltd.

5. Newland, D.E. 1993. An Introduction to Random Vibrations, Spectral and Wavelet analysis. UK: Longman Scientific and Technical, Essex.

6. Bishop, N.W.M. and F. Sherratt, 2000. Finite Element based Fatigue Calculations, UK: NAFEMS Ltd.

7. Bishop, N.W.M. and F. Sherratt, 1989. Fatigue Life Prediction from Power Spectral Density data. Part-1, Traditional Approaches and part-2 Recent Developments. Environ. Eng., 2: 11-19.

8. Bannantine, S.A., J.J. Comer and J.L. Handrock, 1990. Fundamentals of Metal Fatigue Analysis. New Jersey, USA: Prentice-Hall.

9. Bendat, J.S., 1964. Probability functions for random responses. NASA report on Contract NASA-5-4590.

10. Rice, S.O., 1954. Mathematical analysis of random noise. Selected research on Noise and Stochastic Processes. New York: Dover.

11. MSC.FATIGUE, 2005. User's guide. USA: MSC.Software Corporation

12. Tucker, L. and S. Bussa, 1977. The SAE cumulative fatigue damage test program: Fatigue under complex loading, analysis and experiments, Wetzel, R.M. (Ed.). Society of Automotive Engineers, USA, AE-6: 1-54.

13. Felippa, C.A., 2001. Advanced finite element methods. Department of Aerospace Engineering Sciences, University of Colorado.

14. Anthes, R.J. 1997. Modified rainflow counting keeping the load sequences. Inter. J. Fatigue, 19 (7): 529-35.

15. Khosrovaneh, A.K. and N.E. Downing, 1990. Fatigue loading history reconstruction based on rainflow technique. Inter. J. Fatigue, 12 (2): 90-106.

16. Formenti, D., 1999. The relationship between percent of critical and actual damping in a structure. Sound and Vibrations, 33: 14-18.

17. Gade, S., H. Herlufsen and H. Konstantin-Hansen, 2002. How to determine the modal parameters of simple structures. Sound and Vibrations, 36: 72-73.

18. Nastran, M.S.C., 2005. User's guide, Basic dynamic analysis, MSC.Nastran Version 68, MSC. Software Corporation, USA. 